\title{
A Comparative Analysis between Standard and mm-Wave Optimized BEOL in a Nanoscale CMOS Technology
}

\author{
Egidio Ragonese $^{1, *}$ (1) , Claudio Nocera ${ }^{1}$, Andrea Cavarra ${ }^{1}$, Giuseppe Papotto ${ }^{2}$, Simone Spataro ${ }^{1}$ \\ and Giuseppe Palmisano ${ }^{1}$ \\ 1 Dipartimento di Ingegneria Elettrica Elettronica e Informatica (DIEEI), University of Catania, \\ 95125 Catania, Italy; claudio.nocera@unict.it (C.N.); andrea.cavarra@studium.unict.it (A.C.); \\ simone.spataro@phd.unict.it (S.S.); giuseppe.palmisano@unict.it (G.P.) \\ 2 STMicroelectronics, 95121 Catania, Italy; giuseppe.papotto@st.com \\ * Correspondence: egidio.ragonese@unict.it; Tel.: +39-095-738-2331
}

Received: 13 October 2020; Accepted: 8 December 2020; Published: 11 December 2020 updates

\begin{abstract}
This paper presents an extensive comparison of two 28-nm CMOS technologies, i.e., standard and mm-wave-optimized (i.e., thick metals and intermetal oxides) back-end-of-line (BEOL). The proposed comparison is carried out at both component and circuit level by means of a quantitative analysis of the actual performance improvements due to the adoption of a mm-wave-optimized BEOL. To this end, stand-alone transformer performance is first evaluated and then a complete mm-wave macroblock is investigated. A 77-GHz down-converter for frequency modulated continuous wave (FMCW) long-range/medium range (LR/MR) radar applications is exploited as a testbench. For the first time, it is demonstrated that thicker metals and intermetal oxides do not guarantee significant improvements at mm-wave frequencies and a standard (low-cost) BEOL is competitive in comparison with more complex (expensive) ones.
\end{abstract}

Keywords: back-end-of-line (BEOL); CMOS technology; conversion gain; down-converter; electromagnetic simulations; integrated transformers; noise figure; quality factor; radar self-resonance frequency

\section{Introduction}

In recent years, CMOS technologies have reached active device performance comparable to that provided by bipolar processes up to the $W$-band. Moreover, thanks to higher transistor density for analog/digital sections and lower costs for mass-market production, they are an advantageous alternative for the design of a mm-wave system-on-a-chip $(\mathrm{SoC})$ for automotive radar and 5G communications [1]. On the other hand, the improvements in the transition frequency, $f_{\mathrm{T}}$, and maximum oscillation frequency, $f_{\mathrm{MAX}}$, for both MOS and bipolar transistors have been so remarkable that high-frequency IC performance is now mainly limited by the passive component losses (i.e., losses in inductors, transformers, metal interconnections, etc.) [2,3]. In this scenario, a key role is played by the back-end-of-line (BEOL) of the integration process. Traditionally, bipolar and BiCMOS technologies benefit from optimized BEOL for RF/mm-wave application [4-7] with at least two thick copper top metals, along with thick intermetal oxide layers to reduce distributed ohmic losses and parasitic capacitance, respectively. An optimized BEOL is not usually available in standard CMOS technologies, leading to lower quality passive devices. 
However, modern scaled CMOS technologies have demonstrated promising results in comparison with traditional BiCMOS technologies [8]. Figure 1 shows a comparison between a mm-wave-optimized BiCMOS process and a 28-nm CMOS technology with standard BEOL [4,9]. The dilemma is still about the impact of the BEOL on the performance of $\mathrm{mm}$-wave ICs and whether the technological investments can be justified by an effective performance improvement in actual applications. Although several works have faced this issue by comparing stand-alone passive components fabricated in different BEOLs, no analysis on complete mm-wave macroblocks has been published to the authors' knowledge.

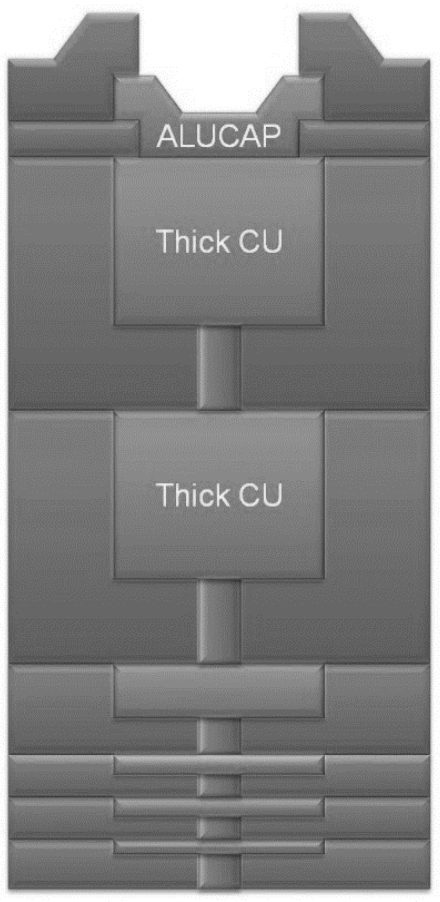

(a)

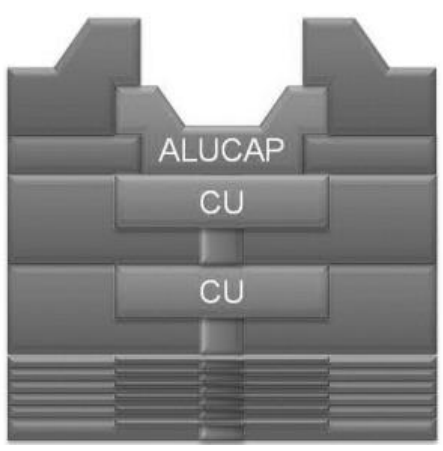

(b)

Figure 1. Back-end-of-line (BEOL) comparison between (a) mm-wave-optimized BiCMOS technology [4] and (b) $28 \mathrm{~nm}$ CMOS technology with standard BEOL [9].

The aim of this paper is to make an extensive comparison between two 28-nm CMOS technologies, i.e., one with a standard BEOL [9] and one with an improved BEOL (i.e., thick metals and oxides), and the ones available in BiCMOS technologies [4-6]. The proposed comparison uses as a demonstrative vehicle a 77-GHz down-converter for radar applications [10], by emphasizing the impact of passive component losses on its overall performance. The choice of this mm-wave macroblock for the proposed comparison is motivated by the stringent and contrasting specifications imposed by radar sensor applications [11,12], in the performance parameters such as noise figure (NF), conversion gain, linearity and power consumption. Indeed, they will be used as a multi-criteria benchmark to highlight the pros and cons of standard and mm-wave oriented CMOS BEOL.

The paper is organized as follows. The adopted testbench for the BEOL comparison is discussed in Section 2. Section 3 deals with the performance comparison of both stand-alone passive components and the overall down-converter, whereas main conclusions are drawn in Section 4.

\section{Testbench for the BEOL Comparison: Integrated Transformers and $77 \mathrm{GHz}$ Down-Converter}

The proposed BEOL comparison was carried out at two different levels, i.e., at both component and circuit level. Initially, the performance of stand-alone integrated transformers designed with different BEOLs is compared, then the analysis is completed by using a 77-GHz down-converter for radar applications, as a macro-circuit testbench. This approach is motivated by the considerations 
discussed below. Integrated transformers are very important passive components, especially at mm-wave frequencies, since they are extensively adopted to implement several crucial functions, such as single-ended-to-differential and differential-to-single-ended conversions along with electrostatic discharge (ESD) protection at input/output antenna interfaces [13-17], resonant load in RF/mm-wave amplification stages [18,19], LC tank in oscillators [20-22], power combining [23,24], etc. Moreover, differently from simple spiral inductors that can highly benefit from top thick metal along with multi-layer structures and maximum oxide thickness to outdistance from the substrate, integrated transformer configurations provide more complete information on the BEOL, since they also include the effects of lower metals and intermetal oxide thickness. Typical performance parameters for the comparison of integrated transformers are the $Q$-factors of the primary and secondary windings, $Q_{\mathrm{P}}$ and $Q_{\mathrm{S}}$, and the magnetic coupling coefficient, $k$. $Q$-factors are directly related to the energy losses occurring in the metal layers that form the transformer coils, as well as in the conductive layers below. They are usually referred to as series and parallel losses, respectively $[25,26]$. The magnetic coupling coefficient measures how much the primary and secondary windings are magnetically coupled to each other and it is mainly dependent on the transformer topology, i.e., interleaved, stacked or mixed topologies with increasing values of $k$ [25-27], as depicted in Figure 2.

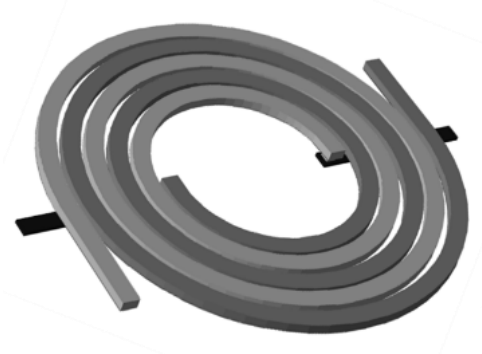

Interleaved

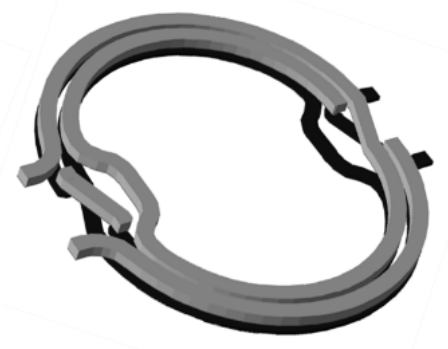

Stacked

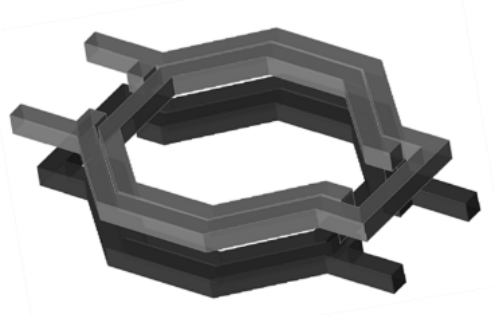

Interstacked

Figure 2. Simplified representation of integrated transformer configurations with increasing magnetic coupling coefficient, $k$.

However, when the transformer is exploited in signal conversion/power combining and resonant load, the insertion loss, $I L$, and the transformer characteristic resistance, TCR, [28] are the more suitable parameters, respectively. Specifically, the $I L$ provides a significant performance characterization when the transformer is operated under a $50-\Omega$ termination at both the primary and secondary windings. Typically, this happens when the transformer is used at the input interface between the antenna and the $\mathrm{RF}$ receiver ( $\mathrm{RX}$ ) front-end (i.e., the RF down-converter). In this situation, the IL directly affects both the gain and NF of the RX front-end. On the other hand, the TCR is the generalization of the well-known $\omega Q L$ product for transformer-loaded circuits. It represents the equivalent impedance offered by the transformer in the resonance condition and is the reference performance parameter when the gain of a transformer-loaded stage has to be maximized. For the sake of completeness, the expressions for $I L$ and $T C R$ are reported below.

$$
\begin{gathered}
I L=-20 \cdot \log S_{21} \\
T C R=\omega Q_{E Q} L_{E Q} \\
Q_{E Q}=Q_{P} \frac{k^{2} Q_{P} Q_{S}}{1+k^{2} Q_{P} Q_{S}} \cong Q_{P} \\
L_{E Q}=L_{P}\left(1+\frac{1}{Q_{P}^{2}}+k^{2} \frac{Q_{S}}{Q_{P}}\right) \cong L_{P}\left(1+k^{2} \frac{Q_{S}}{Q_{P}}\right)
\end{gathered}
$$


where $L_{\mathrm{P}}, Q_{\mathrm{P}}, Q_{\mathrm{S}}$ and $k$ are the primary winding inductance, the primary winding $Q$-factor, the secondary winding $Q$-factor and the magnetic coupling coefficient, respectively.

As far as the 77-GHz down-converter is concerned, the adopted testbench allows a fair comparison between BEOLs since both stacked and interleaved transformer configurations are used, thus providing an overall combined effect at the macroblock level.

\subsection{BEOLs and Integrated Transformers}

The reference CMOS technology is a 28-nm fully-depleted silicon-on-insulator (FD-SOI) CMOS process by STMicroelectronics $[9,29,30]$. It provides a standard BEOL with eight metal layers with two thin $\mathrm{Cu}$ metals (i.e., $<1 \mu \mathrm{m}$ ) plus an $\mathrm{Al}$ layer at the top of the stack, as shown in Figure $1 \mathrm{~b}$. The other technology under comparison differs only for the BEOL, which is oriented to mm-wave applications, as is the one available in SiGe HBT BiCMOS technologies [4]. Specifically, the last two metals are much thicker (around $2.5 \mu \mathrm{m}$ ). Moreover, such a BEOL benefits from a significantly larger distance of the top metal from the silicon substrate compared with the standard one (i.e., about $10 \mu \mathrm{m}$ against $4 \mu \mathrm{m}$ for the top $\mathrm{Al}$ metal layer). For both technologies, the last three metal layers from the top are referred to as LB, IB, and IA.

The last two copper metals, in addition to the aluminum one, were used to design integrated transformers for the $W$-band down-converter with the aim of minimizing the parasitic capacitances towards the substrate and winding resistive losses. In both technologies, transformers adopt octagonal single-turn stacked and interleaved configurations. The primary and secondary windings of the stacked transformers are built in LB/IB and IA respectively, whereas in the interleaved configuration both primary and secondary coils are built in LB/IB, as shown in Figure 3. Due to fabrication issues, the top Al layer, LB, exhibits a hole in transformer coils, as is visible in Figure 3. It is worth mentioning that the adoption of highly involved inter-stacked configurations to maximize the magnetic coupling is mandatory only in presence of very thick intermetal oxides [18,31]. No patterned ground shield (PGS) was included since it would have a detrimental effect on the $Q$-factor at $W$-band frequencies. Indeed, the ohmic losses induced in the shield itself are so high to frustrate the reduction in substrate losses, as described in [32]. Furthermore, avoiding the PGS prevents detrimental resonating phenomena of the shielding structure, mainly due to the undesired inductive path towards the ground in circuit applications. Finally, to avoid additional losses, conductive dummy fillers are not present below the transformer, but only at a safe distance, within a transition region around the spirals, which is sufficiently high to guarantee homogeneity and planarity between metal levels. According to experimental data from the technology provider, such arrangement does not degrade the transformer performance.

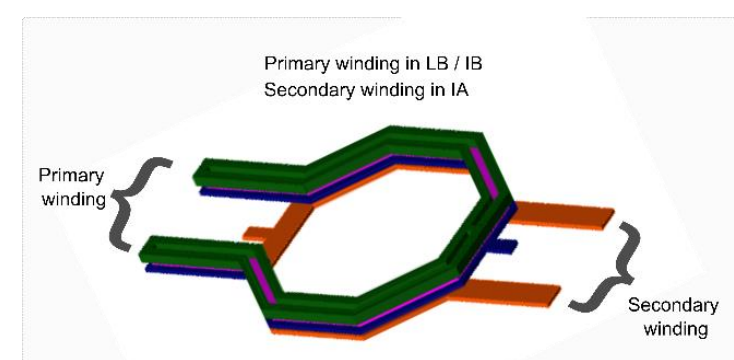

(a)

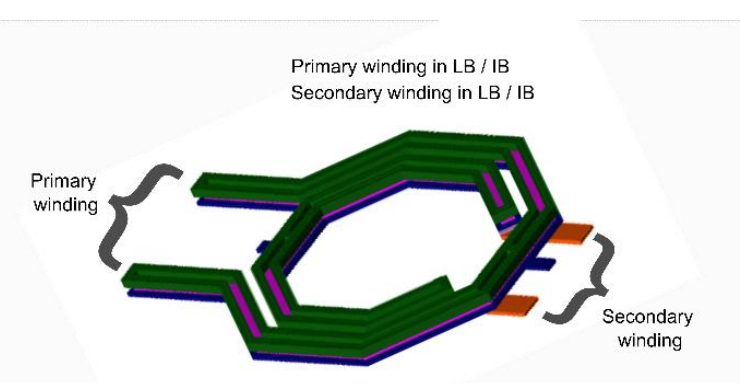

(b)

Figure 3. 3D view of integrated transformers in 28-nm CMOS technology: (a) stacked; (b) interleaved.

As far as geometrical sizing is concerned, transformer windings were designed to achieve a self-resonance frequency $(S R F)$ of about twice the operating frequency (i.e., $77 \mathrm{GHz}$ ). This condition is necessary to guarantee enough room for correct transformer operation in actual applications despite intrinsic and extrinsic parasitic capacitances of active components and interconnections, respectively. 


\section{2. $77 \mathrm{GHz}$ Down-Converter}

The adopted circuit testbench for the BEOL comparison is a $77-\mathrm{GHz}$ direct-conversion down-converter for frequency modulated continuous wave (FMCW) long-range/medium-range (LR/MR) radar applications [33]. This is one of the most critical blocks of a mm-wave radar sensor since it determines the receiver noise/linearity performance, while significantly contributing to its gain. Specifically, in a LR/MR scenario the distance between the sensor and the target, $R$, spans from about $250 \mathrm{~m}$ to $2 \mathrm{~m}$ and thus the power range of the corresponding echo signal is very wide (i.e., -110 to $-30 \mathrm{dBm}$ with typical values of transmitted power, RX/TX antenna gains and radar cross section). Consequently, the radar receiver and hence the mm-wave down-converter, have to achieve a very low NF along with a wide dynamic range (i.e., high-linearity performance). The implementation of a $W$-band down-converter in a nanoscale CMOS technology also has to comply with a supply voltage as low as $1 \mathrm{~V}$, which further exacerbates the linearity issue. Finally, overall performance must be guaranteed at low current consumption since a radar sensor typically adopts a multi receiver architecture [11,12].

A simplified block-diagram of the 77-GHz down-converter used for the BEOL comparison is shown in Figure 4. It exploits a fully differential mixer-first approach consisting of an input transformer, $T_{1}$, a voltage-to-current $(V-I)$ converter, a passive mixer quad and a transresistance feedback amplifier [10]. Transformer $T_{1}$ performs a single-ended-to-differential conversion, ESD protection and $50-\Omega$ input matching. The $V-I$ converter uses transformer $T_{2}$ as its load and achieves a transconductance gain. It drives the mixer, whose output signal current is delivered to the transresistance amplifier. The down-converter design requires careful optimization of both $T_{1}$ and $T_{2}$, whose performances are highly dependent on the available BEOL. Specifically, transformer $T_{1}$ mainly affects the down-converter $N F$ and therefore its $I L$ must be reduced as much as possible. On the other hand, transformer $T_{2}$ can impact the conversion gain if the resonant load impedance of the $V-I$ converter at the operating frequency is not high enough with respect to the mixer input impedance. Indeed, the higher its TCR, the more efficiently the RF current is delivered to the passive mixer, thus preserving the conversion gain.

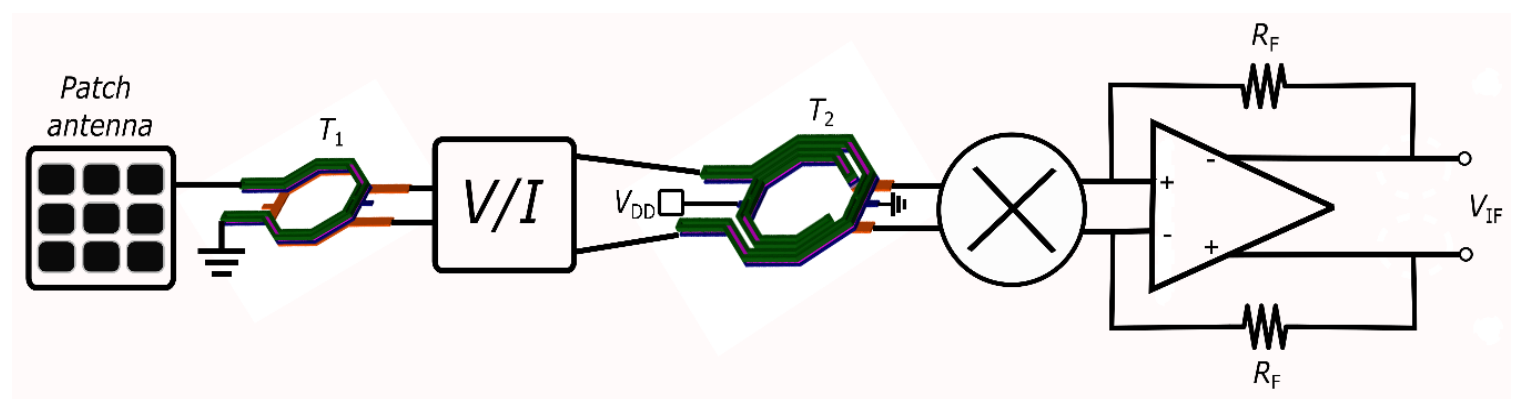

Figure 4. Simplified block-diagram of the down-converter architecture using integrated stacked transformer $\left(T_{1}\right)$ and interleaved $\left(T_{2}\right)$ transformer.

Transformers $T_{1}$ and $T_{2}$ exploit proper configurations (i.e., stacked and interleaved) as shown in Figure 3, to minimize the $I L$ and maximize the TCR, respectively.

\subsection{Experimental Validation for the BEOL Analysis}

Down-converter transformers, $T_{1}$ and $T_{2}$, were designed in both standard- and mm-wave-oriented $\mathrm{BEOL}$, as previously discussed. To this end, extensive 2D electromagnetic (EM) simulations in Advanced Design System (ADS) Momentum by Keysight Technologies were used [34,35], which represent a consolidated design flow for RF and mm-wave ICs, as demonstrated by several published papers in the $K$ - and $W$-bands $[14-18,21]$. To obtain highly reliable results at mm-wave frequencies, the EM simulations required an accurate set-up. The latter was defined and experimentally validated by taking advantage of on-wafer $S$-parameter measurements for a reference stacked transformer, $T_{R}$, 
fabricated in the 28-nm FD-SOI CMOS reference technology [9,30]. Figure 5 displays the micrograph of the ground-signal-ground (GSG) structure adopted for on-wafer measurement of $T_{R}$. It consists of two identical series-connected transformers $T_{R}$, which are put in resonance at $77 \mathrm{GHz}$ by means of an integrated capacitance, $C_{R}$, of about $70 \mathrm{fF}$. This arrangement was exploited to properly draw a meaningful $I L$ measurement of $T_{R}$, since in actual applications it is operated in a resonance condition. As demonstrated in Appendix A, the $I L$ of the single transformer $T_{R}$ can easily be from the $I L$ of the test structure after de-embedding of the GSG pad loss. The comparison between the estimated and measured $I L$ of transformer $T_{R}$ is reported in Figure 6. An excellent agreement can be observed with an error of only $0.3 \mathrm{~dB}$ at $77 \mathrm{GHz}$, which is a very accurate result for such a small component. For the sake of completeness, the measured $R L$ at $77 \mathrm{GHz}$ is about $24 \mathrm{~dB}$, thus confirming the soundness of the adopted extraction method for the $I L$ (see Appendix A). The experimental results for transformer $T_{R}$ were properly used for the EM simulation set-up before designing down-converter transformers $T_{1}$ and $T_{2}$ for the BEOL comparison.

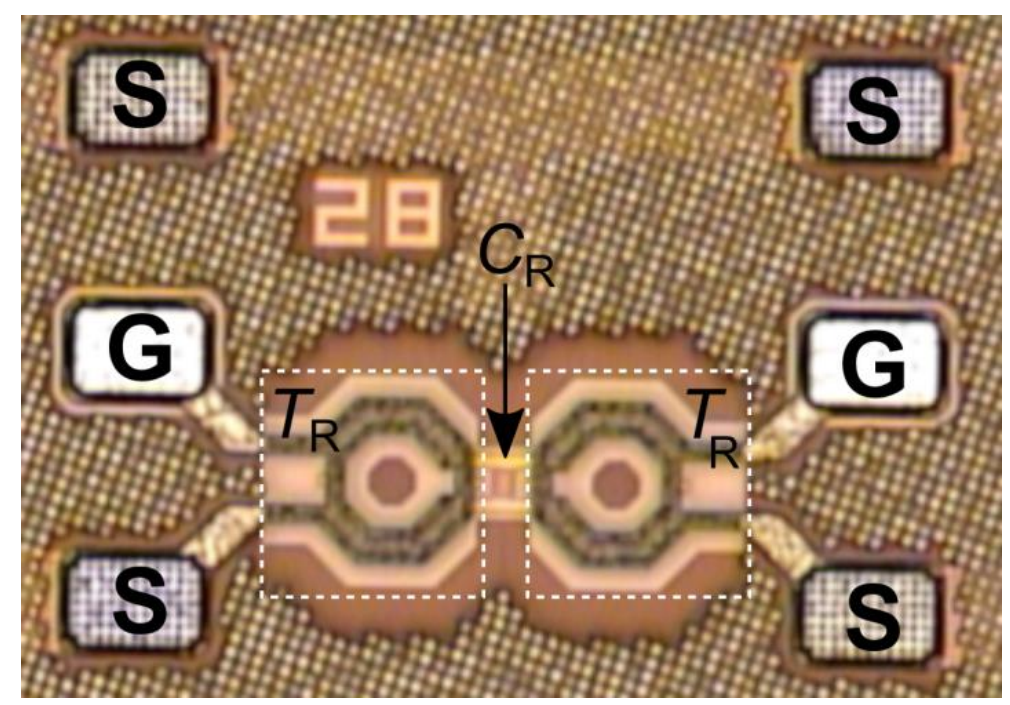

Figure 5. Ground-signal-ground (GSG) structure for on-wafer measurements of transformer $T_{\mathrm{R}}$.

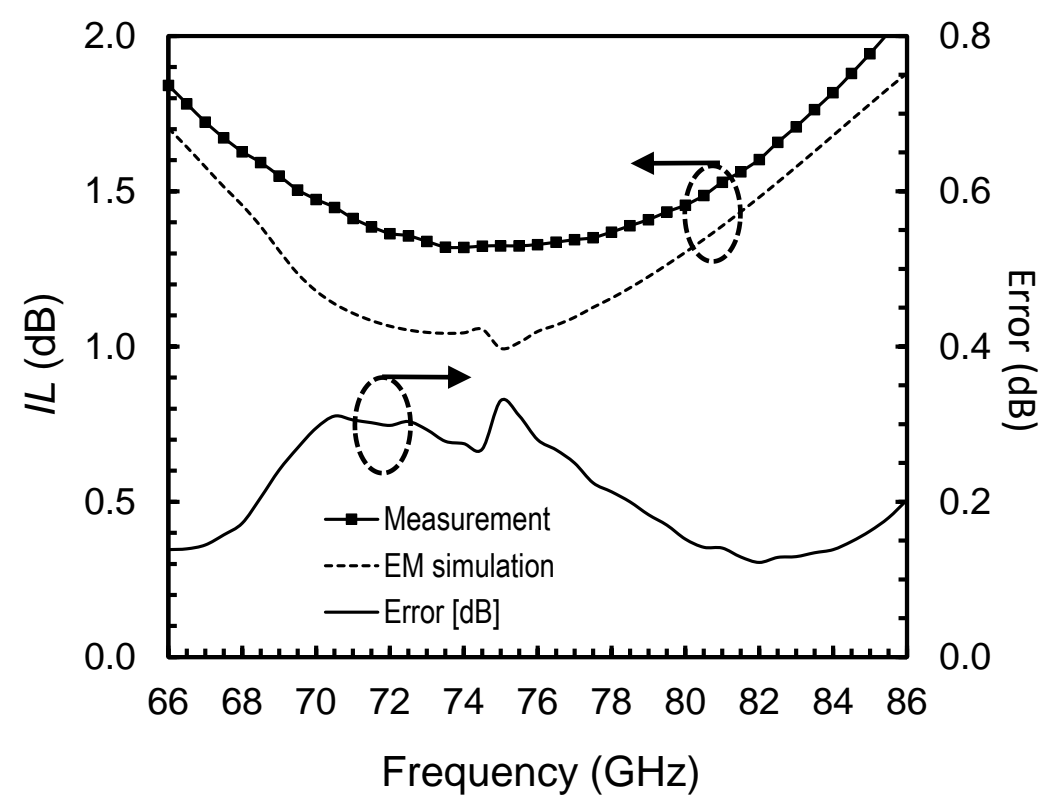

Figure 6. Simulated and measured insertion loss $(I L)$, and corresponding error in $\mathrm{dB}$ of transformer $T_{\mathrm{R}}$. 


\section{BEOL Comparison}

The first step of the BEOL comparison was the geometrical sizing of the down-converter transformers, $T_{1}$ and $T_{2}$, with the aim of optimizing device performance parameters, while guaranteeing the same $S R F$ (i.e., at least two times the operating frequency). Indeed, the simple use of the same transformer geometry in two BEOLs could be not suitable for a fair comparative performance evaluation since the two transformers would have different $S R F$ and hence different levels of optimization. After the stand-alone transformer evaluation in both BEOLs, the comparison was carried out at macroblock level by considering the improvements in terms of $N F$ and conversion gain.

\subsection{Stacked Transformer}

Table 1 reports the geometrical parameters of stacked transformer $T_{1}$, in standard and mm-wave-optimized CMOS BEOL. Main performance parameters, such as winding inductances, $Q$-factors, magnetic coupling coefficient, $k$, insertion loss, $I L$, and transformer characteristic resistance, $T C R$, are compared at the operating frequency of the down-converter (i.e., $77 \mathrm{GHz}$ ). For the sake of completeness, the BEOL comparison in terms of $Q$-factors, $k$ and TCR was also carried out throughout the useful frequency range in Figures 7 and 8 . Several considerations arise from these results.

Table 1. Geometrical and performance parameter comparison for the stacked transformer $T_{1}$.

\begin{tabular}{cccc}
\hline Parameters & $\begin{array}{c}\text { Standard } \\
\text { CMOS BEOL }\end{array}$ & $\begin{array}{c}\text { mm-Wave-Optimized } \\
\text { CMOS BEOL }\end{array}$ & Units \\
\hline Primary/secondary coil width $(w)$ & $5.5 / 5.5$ & $5.5 / 5.5$ & $\mu \mathrm{m}$ \\
Primary/secondary inner diameter $\left(d_{\mathrm{IN}}\right)$ & $44 / 44$ & $60 / 60$ & $\mu \mathrm{m}$ \\
Primary/secondary coil inductance @ 77 GHz & $84 / 96$ & $119 / 130$ & $\mathrm{pH}$ \\
Primary/secondary coil Q-factor @ 77 GHz & $25 / 17$ & $27 / 21$ & - \\
Magnetic coupling factor $(k) @ 77 \mathrm{GHz}$ & 0.63 & 0.62 & $\mathrm{~dB}$ \\
Insertion loss $(I L)$ ) 77 GHz (in resonance) & 1.3 & 1.1 & $\mathrm{k} \Omega$ \\
Transformer characteristic resistance (TCR) @ 77 GHz & 2.3 & 3.6 & $\mathrm{GHz}$ \\
Self-resonance frequency $(S R F)$ & 170 & 170 & \\
\hline
\end{tabular}

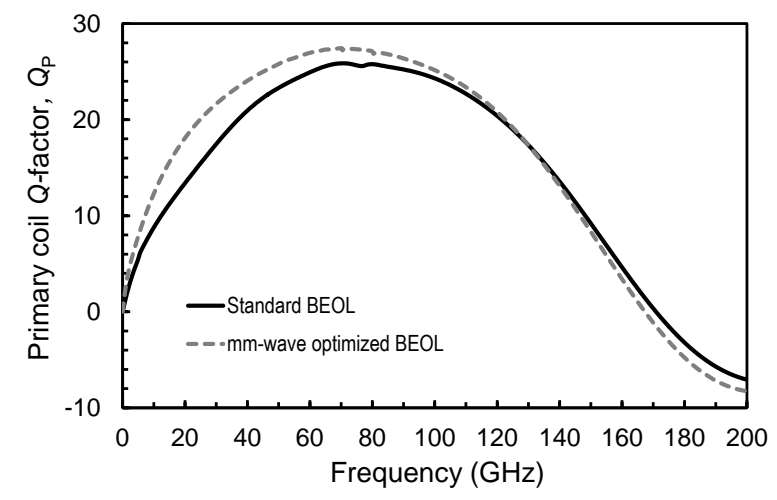

(a)

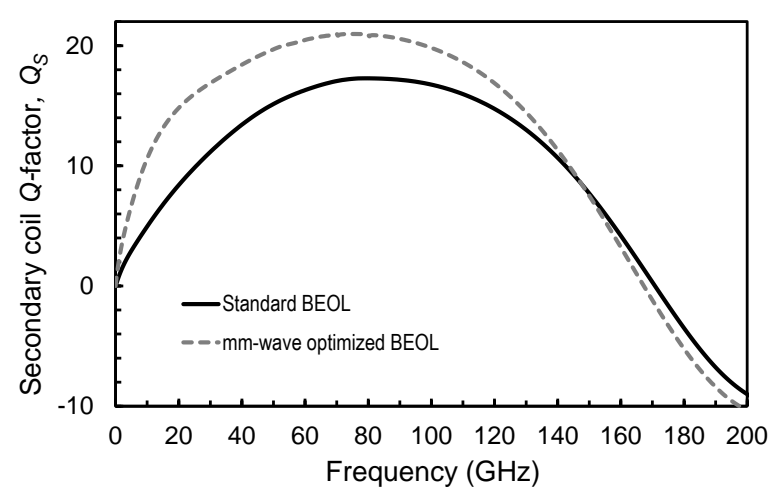

(b)

Figure 7. BEOL comparison for stacked transformer $T_{1}$ of Table 1. (a) primary coil $Q$-factor, $Q_{\mathrm{P}}$; (b) secondary coil $Q$-factor, $Q_{\mathrm{S}}$. 


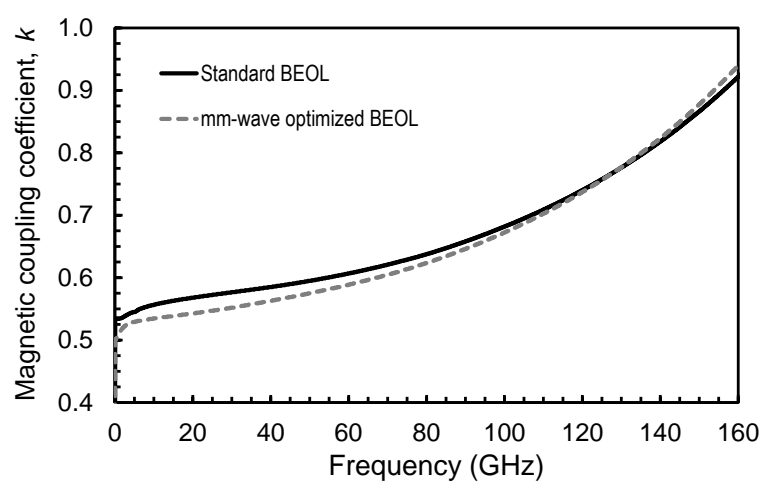

(a)

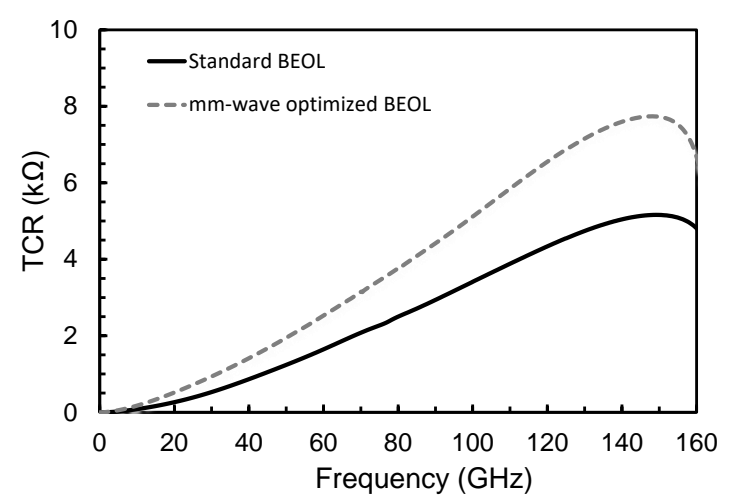

(b)

Figure 8. BEOL comparison for stacked transformer $T_{1}$ of Table 1. (a) magnetic coupling coefficient, $k$; (b) transformer characteristic resistance, TCR.

As already stated, the transformer comparison was carried out at the same $S R F$, which was set equal to $170 \mathrm{GHz}$. In the mm-wave-optimized BEOL, the stacked geometry benefits from a larger inner diameter and hence higher values of primary and secondary coil inductances (of about 42 and 38\% at $77 \mathrm{GHz}$, respectively) thanks to thicker oxide layers and consequent lower parasitic capacitances. Moreover, thanks to thicker top Cu metal layers $(2.5 \mu \mathrm{m}$ instead of $1 \mu \mathrm{m})$ the $Q$-factor of both primary and secondary coils is improved at the cost of a small reduction of $k$ due to the thicker intermetal oxide between windings. In particular, the secondary coil highly benefits from the thick IA Cu layer (see Figure 3a) since its $Q$-factor is improved in a wide operative frequency and achieves an increment of about $23 \%$ at $77 \mathrm{GHz}$, as shown in Figure $7 \mathrm{~b}$. The improvements of the $I L$ and the TCR at $77 \mathrm{GHz}$ are of $0.2 \mathrm{~dB}$ and $56 \%$, respectively, as a direct consequence of higher inductance and $Q$-factor values and an almost constant magnetic coupling, as shown in Figure 8a. The TCR for the optimized BEOL at $77 \mathrm{GHz}$ in Table 1 is comparable with the ones achieved by interleaved configurations in both BEOLs (see next subsection).

\subsection{Interleaved Transformer}

Table 2 reports geometrical and performance parameters of interleaved transformer $T_{2}$ designed in standard and mm-wave-optimized CMOS BEOL, whereas Figures 9 and 10 show the BEOL comparison in terms of $Q$-factors, $k$ and TCR as a function of frequency. Looking at Table 2, it can be noted that, despite the mm-optimized BEOL having thicker oxide layers between the transformer windings (i.e., $\mathrm{AL}+\mathrm{IB}$ ) and the substrate, identical geometrical dimensions (i.e., $w$ and $d_{\mathrm{IN}}$ ) lead to the same $S R F$ (i.e., $175 \mathrm{GHz}$ ). On the other hand, the winding inductance values are slightly lower, i.e., about 8 and $9 \%$ for primary and secondary coils, respectively, due to a higher value of the metal ratio thickness to width (t/w) according to the well-known effect analyzed in [36]. Moreover, there is a negligible increment in both primary and secondary winding $Q$-factors of about $2 \%$, as is also visible in Figure 9 . Consequently, the TCR as defined in Equation (2) results are about the same, as shown in Figure 10b, while the magnetic coupling coefficient, $k$, is slightly improved in the mm-wave-optimized BEOL transformer. These results seem highly counterintuitive since significant improvements in terms of $Q$-factor and TCR would be expected in the best BEOL thanks to thicker metals and oxides. However, the issue should be seen under the correct perspective by starting from the above mentioned $S R F$ invariancy in the interleaved configuration. 
Table 2. Geometrical and performance parameter comparison for the interleaved transformer $T_{2}$.

\begin{tabular}{cccc}
\hline Parameters & $\begin{array}{c}\text { Standard } \\
\text { CMOS BEOL }\end{array}$ & $\begin{array}{c}\text { mm-Wave-Optimized } \\
\text { CMOS BEOL }\end{array}$ & Units \\
\hline Primary/secondary coil width $(w)$ & 5.5 & 5.5 & $\mu \mathrm{m}$ \\
Primary/secondary inner diameter $\left(d_{\mathrm{IN}}\right)$ & $70 / 55$ & $70 / 55$ & $\mu \mathrm{m}$ \\
Primary/secondary coil inductance @ 77 GHz & $130 / 110$ & $120 / 100$ & $\mathrm{pH}$ \\
Primary/secondary coil Q-factor @ 77 GHz & $26 / 22.5$ & $26.5 / 23$ & - \\
Magnetic coupling factor $(k) @ 77 \mathrm{GHz}$ & 0.56 & 0.58 & - \\
Insertion loss $(I L) @ 77 \mathrm{GHz}$ (in resonance) & 1.4 & 1.2 & $\mathrm{~dB}$ \\
Transformer characteristic resistance (TCR) @ 77 GHz & 3.4 & 3.3 & $\mathrm{GHz}$ \\
Self-resonance frequency (SRF) & 175 & 175 & \\
\hline
\end{tabular}

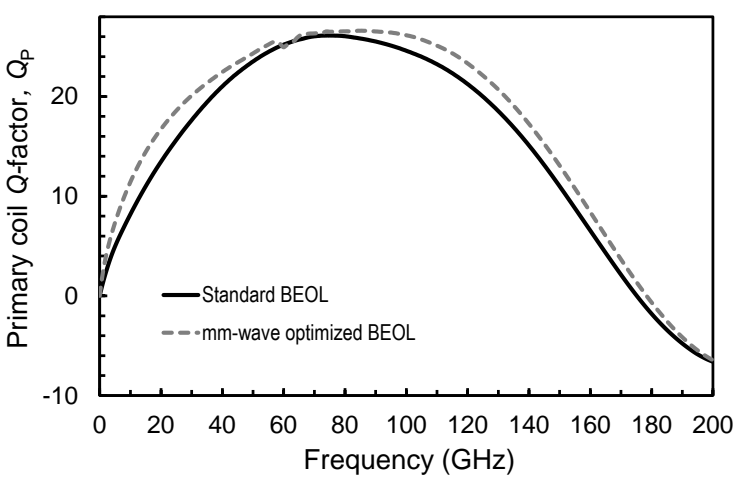

(a)

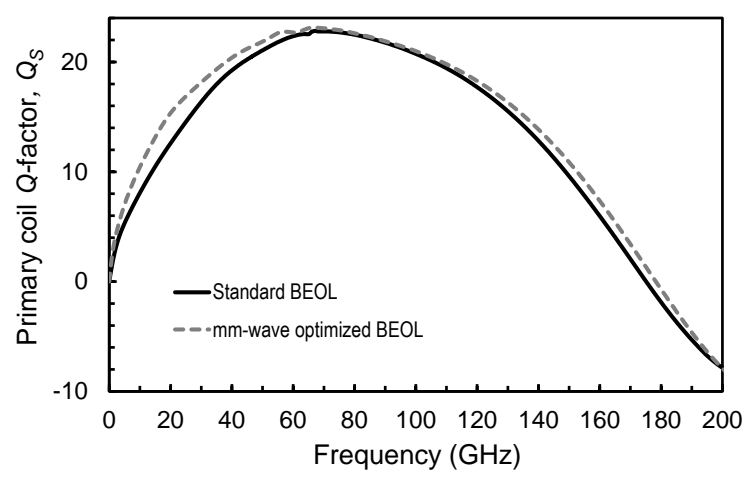

(b)

Figure 9. BEOL comparison for interleaved transformer $T_{2}$ of Table 2: (a) primary coil $Q$-factor, $Q_{\mathrm{P}}$; (b) secondary coil $Q$-factor, $Q_{S}$.

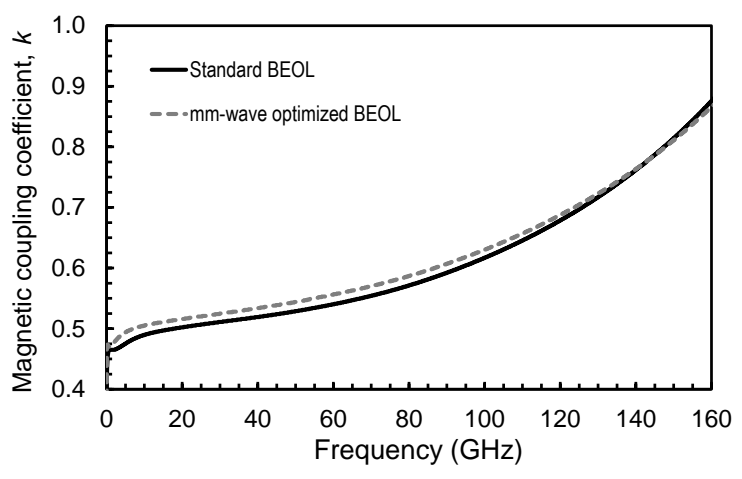

(a)

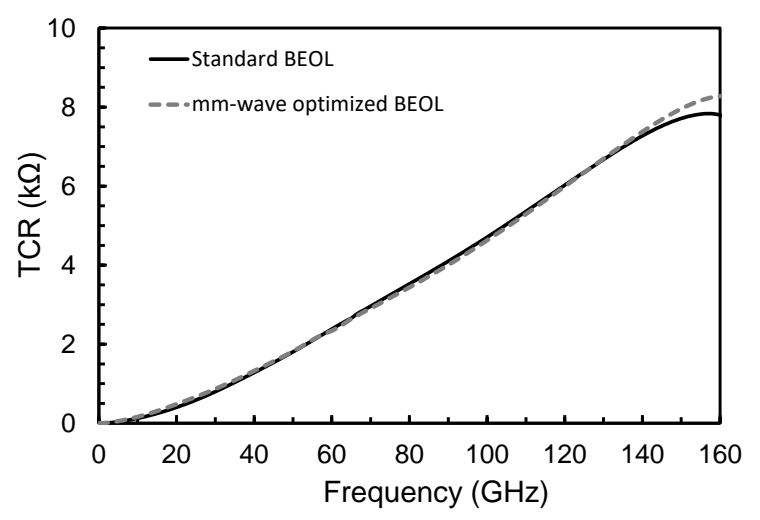

(b)

Figure 10. BEOL comparison for interleaved transformer $T_{2}$ of Table 2: (a) magnetic coupling coefficient $k$; (b) transformer characteristic resistance, TCR.

Let us we consider the well-known equation for a parallel plate capacitor, commonly adopted to evaluate the parasitic capacitances in integrated components:

$$
C=\varepsilon \frac{W \cdot L}{D}=\frac{\varepsilon}{b} L
$$

where $\varepsilon, W, L$ and $D$ are the dielectric permittivity, the width, the length and the distance from the silicon substrate, respectively. The equation can be usefully expressed as a function of the capacitor aspect ratio, $b=D / W$. According to Equation (5), an increased distance of IB from the substrate (from about 3 to $8 \mu \mathrm{m}$ ) would produce a reduction in the transformer capacitance of about 2.5 times with a consequent 
$S R F$ increase of about $60 \%$. On the contrary, an $S R F$ invariancy was detected by EM analysis, as shown in Table 2. This can be mainly ascribed to the very small size of the mm-wave transformers and therefore to the non-negligible effect of the fringing electric field. Indeed, the capacitance of a parallel plate capacitor in Equation (5) was formulated assuming that the separation between the electrode plates is very narrow and then the electric charge density on the plates is uniform, which allows the fringing effects to be neglected. Actually, a crucial role is played by the aspect ratio, $b$. Equation (5) works quite well only for $b<0.03$ with an underestimation of about $10 \%$, whereas for $b \approx 0.5$ it gives errors of about $100 \%$, as depicted in Figure 11 [37,38]. Introducing proper corrections to Equation (5), transformer parasitic capacitances can be better evaluated for the interleaved structure previously analyzed, finding out that the thicker oxide in the mm-wave-optimized BEOL produces a reduction of the capacitance toward the substrate and a contemporary increment of the fringing capacitance of about $75 \%$, which explains the observed $S R F$ invariancy. Therefore, the above analyzed phenomenon tends to frustrate the benefits provided by the optimized BEOL for interleaved transformers. Moreover, this simple analysis can better explain the results in Table 1 and Figures 7 and 8 related to stacked transformers, which highly benefit from the improved BEOL thanks to the lack of significant fringing capacitive effects (i.e., single-turn configuration is used).

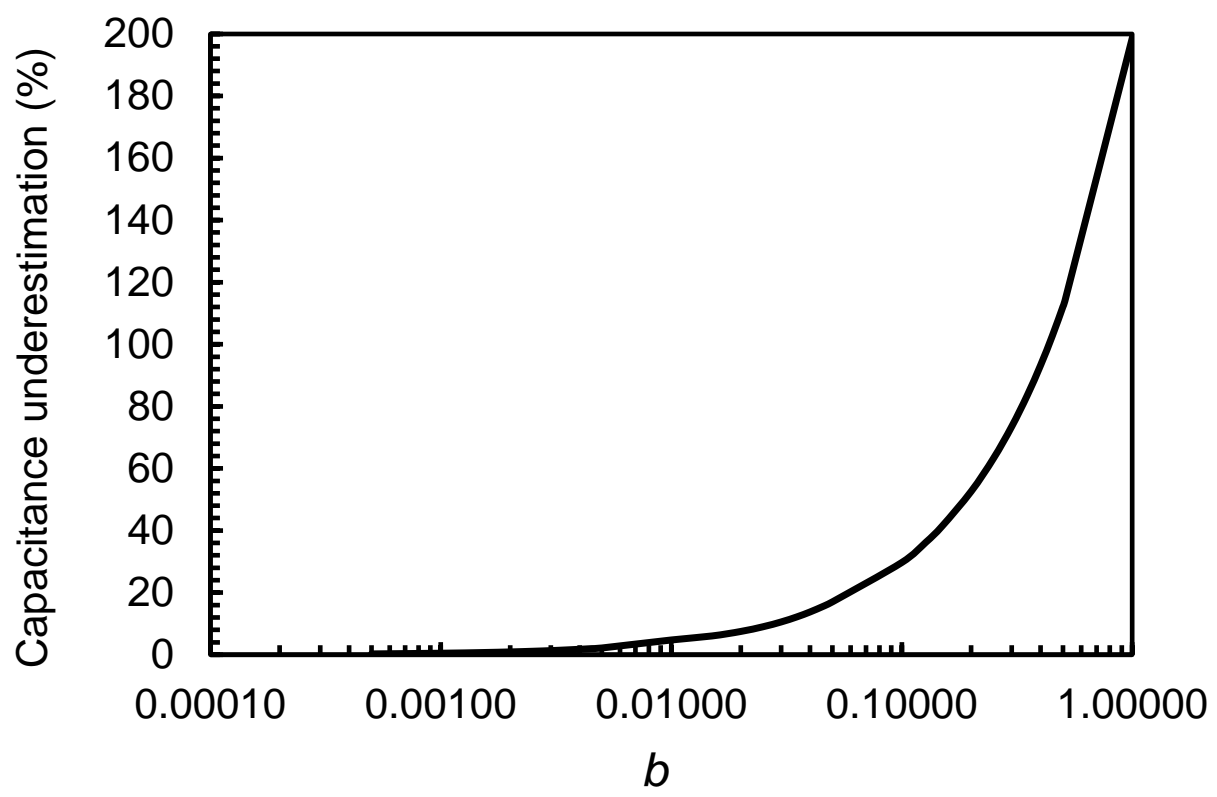

Figure 11. Underestimation of Equation (5) as a function of the aspect ratio, $b$, based on data in [37].

It is worth mentioning that all the above considerations are limited to the analyzed transformer geometries, which are typically adopted at mm-wave frequencies. On the other hand, an improved BEOL could have a very important impact for inductive components operated at lower frequencies where higher inductances and hence larger coils are used, since the percentage effect of the fringing capacitances is greatly reduced.

\section{3. $77 \mathrm{GHz}$ Down-Converter}

Based on transformer performance comparison, a macro-circuit analysis was carried out by exploiting the $77 \mathrm{GHz}$ down-converter architecture for radar applications in Figure 4 [10,30]. Specifically, the BEOL benefits were evaluated in terms of the main performance parameters, i.e., the $N F$ and the conversion gain. As mentioned before, transformers $T_{1}$ and $T_{2}$ exploit stacked and interleaved configurations, respectively, with the aim of minimizing the $I L$ and maximizing the $T C R$, which directly affect the NF and conversion gain, respectively. As far as the $I L$ is concerned, the stacked transformer represents the best solution for the implementation of the input balun in both technologies with values 
of about $0.6 \mathrm{~dB}$ at $77 \mathrm{GHz}$. Concerning the TCR the standard and mm-wave-optimized BEOL provide the highest values with interleaved and stacked configurations, respectively. Nevertheless, to make a meaningful comparison in terms of down-converter performance, the interleaved structure was chosen for the implementation of the inter-stage transformer. This choice is also supported by the very close TCR values (about $3 \mathrm{k} \Omega$ ) achieved by both standard BEOL interleaved and mm-wave-optimized BEOL stacked transformers (see Tables 1 and 2).

The short-circuit transconductance conversion gain and the $N F$ of the $77 \mathrm{GHz}$ down-converter are shown in Figure 12 for both technologies. As expected, the two BEOLs provide similar NF values (around $9 \mathrm{~dB}$ at $1 \mathrm{MHz}$ ), whereas the mm-optimized process allows achievement of a slightly greater conversion gain at $77 \mathrm{GHz}(40.9 \mathrm{mS}$ vs. $40.3 \mathrm{mS})$. Table 3 compares the main performance of the down-converter in the two BEOLs. From these results, it appears that the advantages offered by adopting an optimized BEOL in terms of down-converter performance are almost irrelevant at mm-wave frequencies, as already anticipated by the stand-alone transformer comparison.

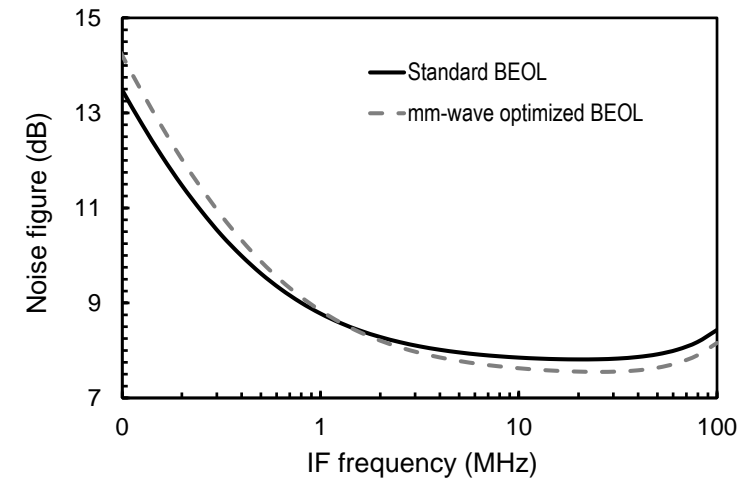

(a)

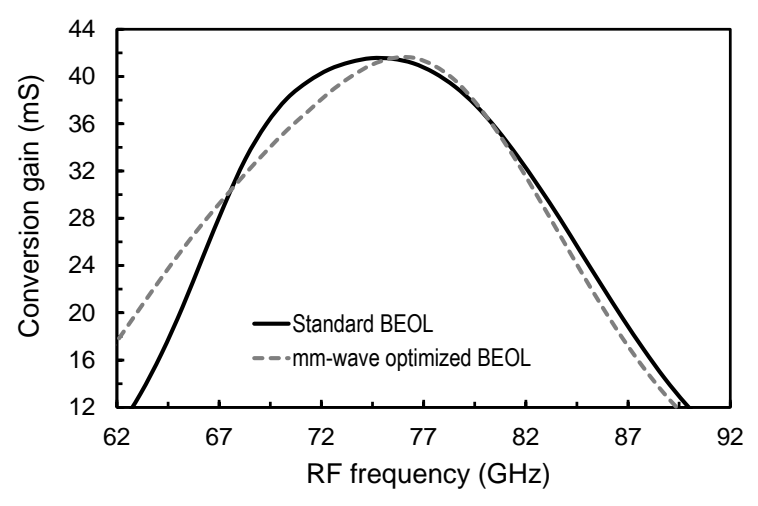

(b)

Figure 12. Down-converter performance: (a) NF as function of the IF frequency; (b) short-circuit transconductance conversion gain as function of the RF frequency.

Table 3. Summary of the $28 \mathrm{~nm}$ CMOS down-converter performance in the two compared BEOLs.

\begin{tabular}{cccc}
\hline Parameters & Standard BEOL & mm-Wave-Optimized BEOL & Units \\
\hline Voltage supply & 1 & 1 & $\mathrm{~V}$ \\
Current consumption & 15 & 15 & $\mathrm{~mA}$ \\
Conversion gain at 77 GHz & 40.3 & 40.9 & $\mathrm{mS}$ \\
NF (IF = 1 MHz) & 9 & 9 & $\mathrm{~dB}$ \\
\hline
\end{tabular}

\section{Conclusions}

Integrated transformers have been designed for a $W$-band down-converter in two 28-nm CMOS technologies, i.e., with a standard and with a mm-wave-optimized BEOL. Despite thicker metals and intermetal oxides providing some advantages, especially for the stacked configuration, mm-wave transformer performance improvements are highly reduced by the EM fringing effects. Indeed, these are not negligible when the transformer size is comparable with the chip vertical dimensions, as happens at $\mathrm{mm}$-wave frequencies. The evaluation of the adopted circuit testcase (i.e., $77 \mathrm{GHz}$ down-converter) further confirms that very limited enhancements can be obtained thanks to an optimized BEOL technology. The reported results question the pros and cons of more expensive and complex BEOLs for nanometer CMOS platforms, providing a different perspective on technology developments for mm-wave applications. 
Author Contributions: Conceptualization, E.R. and G.P. (Giuseppe Papotto); validation, C.N. and A.C.; formal analysis, E.R. and S.S.; investigation, A.C. and C.N.; methodology, E.R. and G.P. (Giuseppe Papotto); project administration, G.P. (Giuseppe Palmisano); supervision, E.R. and G.P. (Giuseppe Papotto); writing-original draft, E.R. and S.S.; writing-review and editing, E.R. All authors have read and agreed to the published version of the manuscript.

Funding: This work was supported in part by the University of Catania through the Project "Programma Ricerca di Ateneo UNICT 2020-22 linea 2".

Acknowledgments: Special thanks are given to A. Castorina and A. Michelin, STMicroelectronics, Catania, for mm-wave measurement assistance.

Conflicts of Interest: The authors declare no conflict of interest.

\section{Appendix A}

Let us consider Figure A1a that shows the equivalent circuit of the test structure in Figure A1a. It consists of two identical series-connected transformers, $T_{R}$, in resonance at $77 \mathrm{GHz}$ by means of capacitor, $C_{R}$. The measured scattering parameter matrix of the test structure in Figure A1a is reported below:

$$
S=\left[\begin{array}{ll}
S_{11} & S_{12} \\
S_{21} & S_{22}
\end{array}\right]=\left[\begin{array}{ll}
S_{11} & S_{21} \\
S_{21} & S_{11}
\end{array}\right]
$$

A further simplification is performed in Figure $\mathrm{A} 1 \mathrm{~b}$ by splitting capacitor $C_{\mathrm{R}}$ to obtain a symmetric cascaded structure $\left(T_{R} / / \frac{C_{R}}{2}\right)$, represented by the corresponding scattering parameter matrix $S^{*}$.

The analysis of the cascade connections of two-port networks can be better carried out by means of the scattering transfer parameters (i.e., chain scattering parameters or T-parameters) [39]:

$$
\left[\begin{array}{l}
a_{1} \\
b_{1}
\end{array}\right]=\left[\begin{array}{ll}
T_{11} & T_{12} \\
T_{21} & T_{22}
\end{array}\right]\left[\begin{array}{l}
b_{2}^{\prime} \\
a_{2}^{\prime}
\end{array}\right]
$$

where $a_{1}, a_{2}^{\prime}$ and $b_{1}, b_{2}^{\prime}$ are the values of the incident and reflected waves at ports 1 and 2, respectively, as shown in Figure A1a. Since in a cascade connection of two-port networks the output waves of the first network are identical to the input waves of the second network i.e., $\left[\begin{array}{c}b_{2} \\ a_{2}\end{array}\right]=\left[\begin{array}{c}a_{1}^{\prime} \\ b_{1}^{\prime}\end{array}\right]$, the T-matrix of the test structure can be written in terms of the individual $T$-matrixes, as follows:

$$
T=T^{*} \cdot T^{*}
$$

The general relationship between $S$ - and $T$-parameters is reported below for both $T$ and $T^{*}$ :

$$
\begin{gathered}
{\left[\begin{array}{ll}
T_{11} & T_{12} \\
T_{21} & T_{22}
\end{array}\right]=\left[\begin{array}{cc}
\frac{1}{S_{21}} & -\frac{S_{22}}{S_{21}} \\
\frac{S_{11}}{S_{21}} & S_{12}-\frac{S_{11} S_{22}}{S_{21}}
\end{array}\right]=\left[\begin{array}{cc}
\frac{1}{S_{21}} & -\frac{S_{11}}{S_{21}} \\
\frac{S_{11}}{S_{21}} & -\frac{S_{11}^{2}-S_{21}^{2}}{S_{21}}
\end{array}\right]} \\
{\left[\begin{array}{ll}
T_{11}^{*} & T_{12}^{*} \\
T_{21}^{*} & T_{22}^{*}
\end{array}\right]=\left[\begin{array}{cc}
\frac{1}{S_{21}^{*}} & -\frac{S_{11}^{*}}{S_{21}^{*}} \\
\frac{S_{11}^{*}}{S_{21}^{*}} & -\frac{\left(S_{11}^{*}\right)^{2}-\left(S_{21}^{*}\right)^{2}}{S_{21}^{*}}
\end{array}\right]}
\end{gathered}
$$

By substituting Equations (A4) and (A5) into Equation (A3) and solving the resulting two-equation system in the unknown $S_{21}^{*}$ and $S_{11}^{*}$ the following relationships can be obtained:

$$
\begin{gathered}
\left(S_{21}^{*}\right)^{2}=S_{21}-\frac{S_{21} S_{11}^{2}}{\left(S_{21}+1\right)^{2}} \\
\left(S_{11}^{*}\right)^{2}=1-\frac{S_{21}}{\left(S_{21}^{*}\right)^{2}}
\end{gathered}
$$


By expressing the modulus of Equation (A7) and neglecting the term $\frac{S_{21} S_{11}^{2}}{\left(S_{21}+1\right)^{2}}$, it is possible to write

$$
\left|S_{21}^{*}\right|=\sqrt{\left|S_{21}-\frac{S_{21} S_{11}^{2}}{\left(S_{21}+1\right)^{2}}\right|} \cong \sqrt{\left|S_{21}\right|}
$$

or equivalently in terms of insertion loss

$$
I L^{*}=\frac{I L}{2}
$$

It is worth noting that assuming Equation (A9) means that the $S_{11}^{*}$ is null or equivalently the return loss, $R L^{*}$, is infinite according to Equation (A7). Therefore, the approximation in Equation (A8) can be done in a narrow frequency band in which the test structure is $50 \Omega$ matched, as shown in Figure 5 .

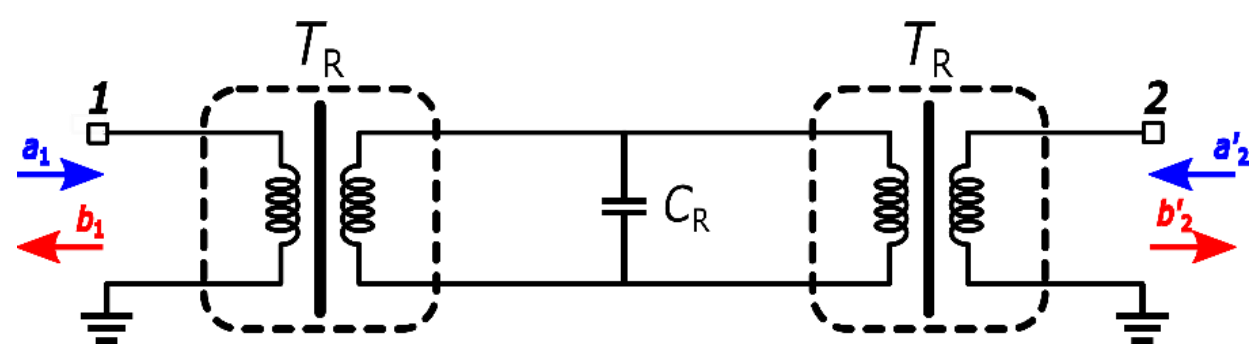

(a)

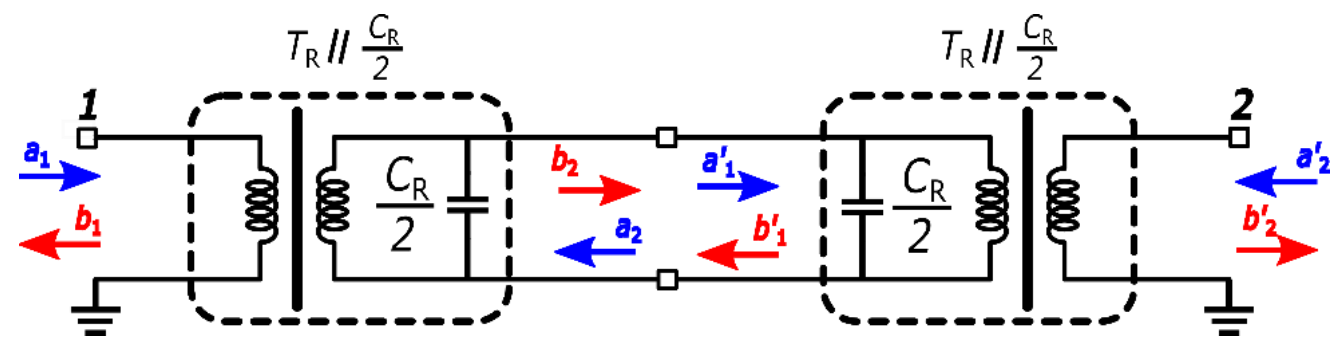

(b)

Figure A1. (a) Equivalent circuit of the transformer test structure in Figure 4a; (b) modified equivalent circuit of the transformer test structure in Figure 4 a.

\section{References}

1. Serhan, A.; Lauga-Larroze, E.; Bourdel, S.; Fournier, J.-M.; Corrao, N. Comparison between MOS and bipolar $\mathrm{mm}$-wave power amplifiers in advanced SiGe technologies. In Proceedings of the IEEE Bipolar/BiCMOS Circuits and Technology Meeting (BCTM), Coronado, CA, USA, 28 September-1 October 2014; pp. 159-162.

2. Chevalier, P.; Liebl, W.; Rucker, H.; Gauthier, A.; Manger, D.; Heinemann, B.; Avenier, G.; Bock, J. SiGe BiCMOS Current Status and Future Trends in Europe. In Proceedings of the 2018 IEEE BiCMOS and Compound Semiconductor Integrated Circuits and Technology Symposium (BCICTS), San Diego, CA, USA, 15-17 October 2018; pp. 64-71.

3. Spasaro, M.; Zito, D. Millimeter-Wave Integrated Silicon Devices: Active versus Passive-The Eternal Struggle Between Good and Evil: (Invited Paper). In Proceedings of the 2019 International Semiconductor Conference (CAS), Sinaia, Romania, 9-11 October 2019; pp. 11-20.

4. Avenier, G.; Diop, M.; Chevalier, P.; Troillard, G.; Loubet, N.; Bouvier, J.; Depoyan, L.; Derrier, N.; Buczko, M.; Leyris, C.; et al. $0.13 \mathrm{~m}$ SiGe BiCMOS Technology Fully Dedicated to mm-Wave Applications. IEEE J. Solid State Circuits 2009, 44, 2312-2321. [CrossRef] 
5. Bock, J.; Aufinger, K.; Boguth, S.; Dahl, C.; Knapp, H.; Liebl, W.; Manger, D.; Meister, T.F.; Pribil, A.; Wursthorn, J.; et al. SiGe HBT and BiCMOS process integration optimization within the DOTSEVEN project. In Proceedings of the 2015 IEEE Bipolar/BiCMOS Circuits and Technology Meeting-BCTM, Boston, MA, USA, 26-28 October 2015; pp. 121-124.

6. Pekarik, J.J.; Adkisson, J.; Gray, P.; Liu, Q.; Camillo-Castillo, R.; Khater, M.; Jain, V.; Zetterlund, B.; Divergilio, A.; Tian, X.; et al. A $90 \mathrm{~nm}$ SiGe BiCMOS technology for mm-wave and high-performance analog applications. In Proceedings of the 2014 IEEE Bipolar/BiCMOS Circuits and Technology Meeting (BCTM), Coronado, CA, USA, 28 September-1 October 2014; pp. 92-95.

7. Chevalier, P.; Avenier, G.; Ribes, G.; Montagné, A.; Canderle, E.; Céli, D.; Derrier, N.; Deglise, C.; Durand, C.; Buczko, M.; et al. A $55 \mathrm{~nm}$ triple gate oxide 9 metal layers SiGe BiCMOS technology featuring $320 \mathrm{GHz}$ $\mathrm{f}_{\mathrm{T}} / 370 \mathrm{GHz} \mathrm{f}_{\mathrm{MAX}} \mathrm{HBT}$ and high-Q millimeter-wave passives. In Proceedings of the IEEE International Electron Devices Meeting, San Francisco, CA, USA, 15-17 December 2014; pp. 3.9.1-3.9.3.

8. Joseph, A.; Jain, V.; Ong, S.N.; Wolf, R.; Lim, S.F.; Singh, J. Technology positioning for mm-wave applications: 130/90nm SiGe BiCMOS vs. 28nm RFCMOS. In Proceedings of the IEEE BiCMOS and Compound Semiconductor Integrated Circuits and Technology Symposium (BCICTS), San Diego, CA, USA, 15-17 October 2018; pp. 18-21.

9. Cathelin, A. Fully Depleted Silicon on Insulator Devices CMOS: The 28-nm Node Is the Perfect Technology for Analog, RF, mmW, and Mixed-Signal System-on-Chip Integration. IEEE Solid State Circuits Mag. 2017, 9, 18-26. [CrossRef]

10. Nocera, C.; Cavarra, A.; Ragonese, E.; Palmisano, G.; Papotto, G. Down-converter solutions for 77-GHz automotive radar sensors in 28-nm FD-SOI CMOS technology. In Proceedings of the 2018 14th Conference on Ph.D. Research in Microelectronics and Electronics (PRIME), Prague, Czech Republic, 2-5 July 2018; pp. 153-156.

11. Fujibayashi, T.; Takeda, Y.; Wang, W.; Yeh, Y.-S.; Stapelbroek, W.; Takeuchi, S.; Floyd, B. A 76- to 81-GHz Multi-Channel Radar Transceiver. IEEE J. Solid State Circuits 2017, 52, 2226-2241. [CrossRef]

12. Trotta, S.; Wintermantel, M.; Dixon, J.; Moeller, U.; Jammers, R.; Hauck, T.; Samulak, A.; Dehlink, B.; Shun-Meen, K.; Li, H.; et al. An RCP Packaged Transceiver Chipset for Automotive LRR and SRR Systems in SiGe BiCMOS Technology. IEEE Trans. Microw. Theory Tech. 2012, 60, 778-794. [CrossRef]

13. Raczkowski, J.; Thijs, S.; De Raedt, W.; Nauwelaers, B.; Wambacq, P. 50-to-67GHz ESD-protected power amplifiers in digital 45nm LP CMOS. In Proceedings of the 2009 IEEE International Solid-State Circuits Conference-Digest of Technical Papers, San Francisco, CA, USA, 8-12 February 2009; pp. 382-383.

14. Giammello, V.; Ragonese, E.; Palmisano, G. A 15-dBm SiGe BiCMOS PA for 77-GHz Automotive Radar. IEEE Trans. Microw. Theory Tech. 2011, 59, 2910-2918. [CrossRef]

15. Ragonese, E.; Giammello, V.; Palmisano, G. A 24/77-GHz SiGe BiCMOS transmitter chipset for automotive radar. Wiley Microw. Opt. Technol. Lett. 2013, 55, 782-786.

16. Scuderi, A.; Ragonese, E.; Palmisano, G. 24-GHz ultra-wideband transmitter for vehicular short-range radar applications. IET Circuits Devices Syst. 2009, 3, 313-321. [CrossRef]

17. Ragonese, E.; Scuderi, A.; Giammello, V.; Palmisano, G. A SiGe BiCMOS 24-GHz receiver front-end for automotive short-range radar. Analog. Integr. Circuits Signal Process. 2010, 67, 121-130. [CrossRef]

18. Giammello, V.; Ragonese, E.; Palmisano, G. A Transformer-Coupling Current-Reuse SiGe HBT Power Amplifier for 77-GHz Automotive Radar. IEEE Trans. Microw. Theory Tech. 2012, 60, 1676-1683. [CrossRef]

19. Giammello, V.; Ragonese, E.; Palmisano, G. Transformer-coupled cascode stage for mm-wave power amplifiers in sub- $\mu \mathrm{m}$ CMOS technology. Analog. Integr. Circuits Signal Process. 2010, 66, 449-453. [CrossRef]

20. Ragonese, E.; Scuderi, A.; Biondi, T.; Palmisano, G. Scalable lumped modeling of single-ended and differential inductors for RF IC design. Int. J. RF Microw. Comput. Eng. 2009, 19, 110-119. [CrossRef]

21. Sapone, G.; Ragonese, E.; Italia, A.; Palmisano, G. A 0.13- $\mu \mathrm{m}$ SiGe BiCMOS colpitts-based VCO for W-band radar transmitters. IEEE Trans. Microw. Theory Tech. 2013, 61, 185-194. [CrossRef]

22. Kashani, M.H.; Tarkeshdouz, A.; Molavi, R.; Sheikholeslami, A.; Afshari, E.; Mirabbasi, S. On the Design of a High-Performance mm-Wave VCO With Switchable Triple-Coupled Transformer. IEEE Trans. Microw. Theory Tech. 2019, 67, 4450-4464. [CrossRef]

23. An, K.H.; Lee, O.; Kim, H.; Lee, N.H.; Han, J.; Yang, K.S.; Kim, Y.; Chang, J.J.; Woo, W.; Lee, C.-H.; et al. Power-Combining Transformer Techniques for Fully-Integrated CMOS Power Amplifiers. IEEE J. Solid State Circuits 2008, 43, 1064-1075. [CrossRef] 
24. Sandstrom, D.; Martineau, B.; Varonen, M.; Karkkainen, M.; Cathelin, A.; Halonen, K.A.I. 94 GHz power-combining power amplifier with $+13 \mathrm{dBm}$ saturated output power in $65 \mathrm{~nm}$ CMOS. In Proceedings of the IEEE Radio Frequency Integrated Circuits Symposium, Baltimore, MD, USA, 5-7 June 2011; pp. 1-4.

25. Scuderi, A.; Ragonese, E.; Biondi, T.; Palmisano, G. Integrated Inductors and Transformers: Characterization, Design and Modeling for Rf and Mm-Wave Applications; CRC Press-Taylor Francis Group: Boca Raton, FL, USA, 2010.

26. Spataro, S.; Ragonese, E. Design and optimization of silicon-integrated inductive components for automotive radar applications in $\mathrm{K}$ - and $\mathrm{W}$-bands. In Proceedings of the 2020 AEIT International Conference of Electrical and Electronic Technologies for Automotive (AEIT AUTOMOTIVE), Torino, Italy, 18-20 November 2020; pp. 1-6.

27. Ragonese, E.; Sapone, G.; Palmisano, G. High-performance interstacked transformers for mm-wave ICs. Microw. Opt. Technol. Lett. 2010, 52, 2160-2163. [CrossRef]

28. Carrara, F.; Italia, A.; Ragonese, E.; Palmisano, G. Design methodology for the optimization of transformer-loaded RF circuits. IEEE Trans. Circuits Syst. I Regul. Pap. 2006, 53, 761-768. [CrossRef]

29. Larie, A.; Kerhervé, E.; Martineau, B.; Vogt, L.; Belot, D. A 60 GHz 28 nm UTBB FD-SOI CMOS reconfigurable power amplifier with 21\% PAE, $18.2 \mathrm{dBm}$ P1 dB and $74 \mathrm{~mW}$ PDC. In Proceedings of the 2015 IEEE International Solid-State Circuits Conference-(ISSCC) Digest of Technical Papers, San Francisco, CA, USA, 22-26 February 2015; pp. 1-3.

30. Cavarra, A.; Nocera, C.; Papotto, G.; Ragonese, E.; Palmisano, G. Transformer design for 77-GHz down-converter in 28-nm FD-SOI CMOS Technology. In Lecture Notes in Electrical Engineering, Proceedings of the International Conference on Applications in Electronics Pervading Industry, Environment and Society, Pisa, Italy, 26-27 September 2018; Springer: New York, NY, USA, 2018; pp. 195-201.

31. Ragonese, E.; Sapone, G.; Giammello, V.; Palmisano, G. Analysis and modeling of interstacked transformers for mm-wave applications. Analog. Integr. Circuits Signal Process. 2011, 72, 121-128. [CrossRef]

32. Spataro, S.; Salerno, N.; Papotto, G.; Ragonese, E. The effect of a metal PGS on the Q-factor of spiral inductors for RF and mm-wave applications in a 28-nm CMOS technology. Int. J. RF Microw. Comput. Eng. 2020, 30, 1-12. [CrossRef]

33. Saponara, S.; Greco, M.; Ragonese, E.; Palmisano, G.; Neri, B. Highly Integrated Low Power Radars; Artech House: London, UK, 2014; ISBN 9781608076659.

34. Keysight Technologies. Momentum 3D Planar EM Simulator. Available online: https://www.keysight.com/ en/pc-1887116/momentum-3d-planar-em-simulator?cc=US\&lc=eng (accessed on 9 December 2020).

35. Ragonese, E.; Palmisano, G. Design of a SiGe BiCMOS low-noise amplifier for 24-GHz UWB automotive radar. In Proceedings of the European Agilent Technologies ADS Users' Group Meeting, Rome, Italy, 13 May 2009. Available online: http://www.home.agilent.com/upload/cmc_upload/All/RagoneseEuropeanADSMeetingweb. pdf (accessed on 9 December 2020).

36. Scuderi, A.; Biondi, T.; Ragonese, E.; Palmisano, G. Analysis and modeling of thick-metal spiral inductors on silicon. In Proceedings of the 2005 European Microwave Conference, Paris, France, 4-6 October 2005; pp. 81-84.

37. Nishiyama, H.; Nakamura, M. Capacitance of a strip capacitor. IEEE Trans. Compon. Hybrids Manuf. Technol. 1990, 13, 417-423. [CrossRef]

38. Poh, S.Y.; Chew, W.C.; Kong, J.A. Approximate formulas for line capacitance and characteristic impedance of microstrip line. IEEE Trans. Microw. Theory Tech. 1981, 29, 135-142. [CrossRef]

39. Gonzales, A. Microwave Transistor Amplifiers: Analysis and Design, 2nd ed.; Prentice Hall: Upper Saddle River, NJ, USA, 1984; pp. 8-12.

Publisher's Note: MDPI stays neutral with regard to jurisdictional claims in published maps and institutional affiliations.

(C) 2020 by the authors. Licensee MDPI, Basel, Switzerland. This article is an open access article distributed under the terms and conditions of the Creative Commons Attribution (CC BY) license (http://creativecommons.org/licenses/by/4.0/). 